\title{
Mobile computing based on GSM: The Mowgli approach
}

\author{
Timo Alanko, Markku Kojo, Heimo Laamanen, Kimmo Raatikainen, \\ and Martti Tienari \\ University of Helsinki \\ Department of Computer Science, P.O. Box 26 (Teollisuuskatu 23), \\ FIN-00014 University of Helsinki, Finland. \\ Telephone: +358-0-70851. Fax: +358-0-70844441. \\ email: \{timo.alanko,markku.kojo, heimo.laamanen, \\ kimmo.raatikainen, martti.tienari\}@cs.Helsinki.FI
}

\begin{abstract}
Modern cellular telephone systems extend the usability of portable personal computers enormously. A nomadic user can be given ubiquitous access to remote information stores and computing services. However, the behavior of wireless links creates severe inconveniences within the traditional data communication paradigm. In this paper we give an overview of the problems related to wireless mobility. We also present a new software architecture for mastering them and discuss a new paradigm for designing mobile distributed applications. The key idea in the architecture is to place a mediator, a distributed intelligent agent, between the mobile node and the wireline network. A prototype implementation of the architecture exists in an environment consisting of Linux and Windows platforms and the GSM cellular telephone network.
\end{abstract}

\section{Keywords}

Mobile clients, intelligent agents, nomadic users, personal communications, GSM Data Service.

\section{INTRODUCTION}

Developments in mobile communication and personal computer technology have created a new interesting platform for information processing. A modern portable computer gives remarkable processing power, always at hand for a nomadic user. A mobile telephone system gives ubiquitous access to remote information stores and computing services.

One of the most widely available cellular telephone systems is the Global System for Mobile Communications (GSM); see Rahnema (1993). It covers vast areas in Europe, and it is rapidly expanding in Asia and in Australia. It is also becoming available in the US. In addition, other cellular telephone systems already exist, and still more are emerging. This 
development implies a tremendous increase in the accessibility of information services. In principle, a mobile computer can always be connected to its home network through a cellular telephone system. Thus, the users will have the impression of working with their "normal desktop computers" wherever they happen to be. In practice, however, several reasons still prevent this scenario from working to the full satisfaction of the user. The problems are due to the different natures of the wireline and wireless worlds: the former is efficient and reliable, the latter is slow and vulnerable. Control methods tuned for one are not necessarily suitable for the other.

In Kojo et al. (1994) we introduced the Mowgli* approach to alleviate these problems. The key idea is to separate the behaviorally different wireline and wireless worlds. On the border of these two worlds we add a new component, a mediator. It contains intelligence, and it is able to cope with both worlds. In essence, this is a shift of paradigm: the traditional "client-server" paradigm is replaced with a new "client-mediator-server" paradigm.

At the same time similar principles were independently introduced for wireless LANs; see Yavatkar and Bhagawat (1994), Bakre and Badrinath (1995), Balakrishnan et al. (1995). However, in the wireless LAN the problem is treated at the transport layer. In Mowgli it is seen as a problem concerning all layers up to the application layer and the user interface.

In Section 2 we address the fundamental problems related to mobility and wirelessness and in Section 3 we outline our mediator-based Mowgli solution. The benefits of Mowgli are discussed in Section 4. In Section 5 we summarize the essentials of the Mowgli approach.

\section{PROBLEMS RELATED TO MOBILITY AND WIRELESSNESS}

Under favorable radio conditions a wireless telephone link behaves almost like a traditional PSTN link. Therefore, it can be expected that existing applications still work when the conditions are good. However, there are differences between wireline and wireless links that must be taken into account:

- Wireless telephone links have low throughput, high latency, and a long connection establishment time.

- Wireless links are expensive. Today, the GSM offers only connection oriented services. Thus, customary habits of working with the link open over the whole session are not economical.

- Transmission delays may be highly variable. When error rates on radio links are high, the strong error correction mechanisms of the GSM data service increase significantly the delay variation. In unfavorable conditions the delays may extend to tens of seconds.

- The wireless link is vulnerable. The mobile workstation may move through an uncovered area, or the radio conditions may temporarily deteriorate. In both cases the link becomes non-accessible for some period of time.

Current communication architectures, such as the TCP/IP protocol suite, usually offer improper performance over networks with wireless links. In addition, neither they nor the

\footnotetext{
${ }^{*}$ Mowgli is the acronym of the project name Mobile Office Workstations using GSM Links.
} 


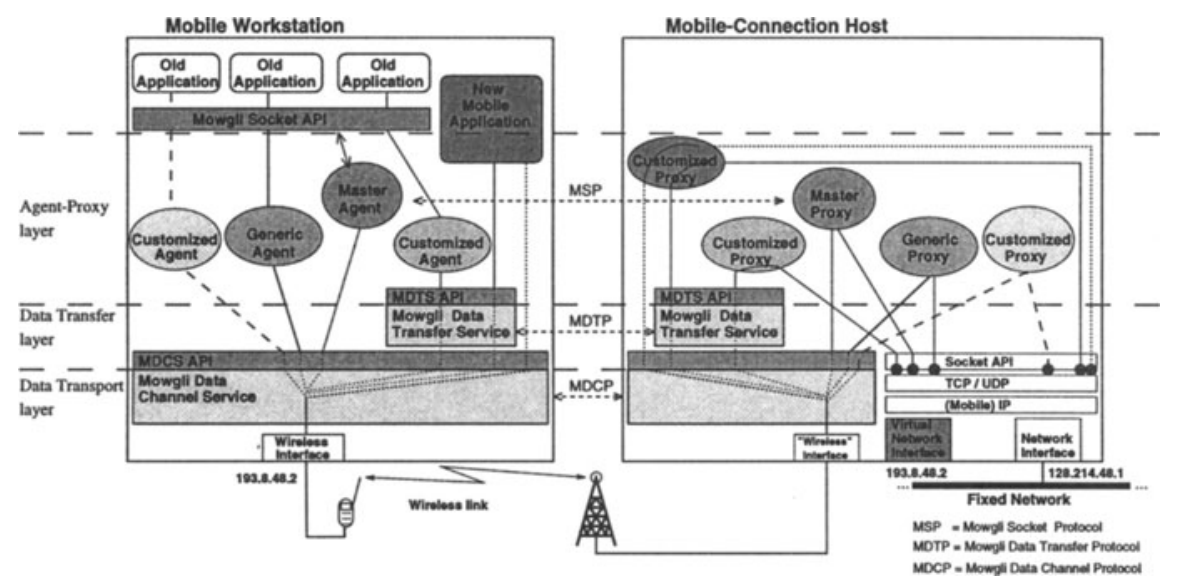

Figure 1 The Mowgli communication architecture for connecting mobile nodes to fixed networks

existing applications are designed to react in an appropriate way to the behavior characteristics of wireless WAN environments. Hence, even minor disturbances in connections usually result in failures at the application level as explained in Kojo et al. (1994) and in Alanko et al. (1994).

The user confronted with these circumstances would benefit from having new functionalities provided by the infrastructure: information about expected performance, direct control over the transfer operations, possibility to specify a condition-based control policy, capability to work in disconnected mode, advanced error recovery methods.

\section{A MEDIATOR-BASED APPROACH: THE MOWGLI COMMUNICATION ARCHITECTURE}

The key idea in the Mowgli architecture is to separate the behaviorally different wireline and wireless worlds. The essential component for doing this is the mediator, a node located in the fixed network.

A mobile node is connected to the fixed network through a wireless telephone link. The mediator is the node which provides the mobile node with a connection point to the wireline Internet. In Mowgli the mediator node is called the mobile-connection host (MCH).

The Mowgli system offers to the applications on the mobile node a rich set of communication and control services. Figure 1 depicts the conceptual organization of the service architecture. Services are provided in three different layers: in the agent-proxy layer, in the data transfer layer, and in the data transport layer.

The basic communication services are available through an API called the Mowgli socket interface. This interface includes the socket operations of the traditional BSD socket interface. Hence, existing applications that use TCP or UDP sockets can be executed on 
the mobile node without modifications. In addition to this, the Mowgli sockets provide new features not available in conventional TCP/IP sockets. These features include assignment of priorities to the data delivered through the socket, establishing the wireless link, and ordering an automatic recovery from unexpected link-level disconnections.

The Mowgli socket interface binds the existing applications to the services available in the agent-proxy layer. These services are implemented as agent-proxy teams. A proxy on the MCH and an agent on the mobile node cooperate to act as the mediator for all the data delivered between an application on the mobile node and its peer in the Internet. The proxy has a special mission: it plays the role of the mobile node while communicating on its behalf with the peer.

In the agent-proxy layer we have three different kinds of teams:

1. Master agent - Master proxy. Master agent and proxy provide the semantics of the traditional socket operations. In order to accomplish this the Mowgli socket layer informs the master agent whenever an application on the mobile node invokes a socket operation. The master agent then sends a message to the master proxy which performs the corresponding operation on behalf of the application. The masters are able to create other agents and proxies. They also take care of delivering UDP datagrams.

2. Generic agent - Generic proxy. Once a TCP connection with a remote peer has been established on a TCP socket the masters create a generic agent-proxy team, which will take care of delivering the data for that socket.

3. Customized agent - Customized proxy. The generic agent and proxy can be replaced with a customized agent and proxy which are tailored for a specific application protocol, for example for the HTTP protocol used in the World-Wide Web. The Mowgli WWW software, see Liljeberg et al. $(1995,1996)$, is a good example of an agent-proxy team taking advantage of its knowledge about application semantics.

The functionality of a customized agent can be integrated into the client software when new applications are implemented for mobile users. Such a mobile client can cooperate directly with the customized proxy on the $\mathrm{MCH}$. This approach amounts to splitting the traditional client program into two parts - one part on each side of the wireless link. The communication services available in the data transfer layer and in the data transport layer are specifically designed to support application-specific agent-proxy teams to be used in communication over the wireless (telephone) link.

In the data transfer layer, the Mowgli Data Transfer Service (MDTS) is able to take over the responsibility of transferring structured user data over the wireless link. The basic element of information for transfer operations is Information eXchange Unit (IXU). In general, an IXU is something that the user considers as an independent unit of information, the transfer of which he or she may want to control. Examples of IXUs include mail messages, files, print jobs, and inline images of WWW pages. Each IXU can have a set of attributes, which are used in controlling the transfer of the IXU. The MDTS provides an API for invoking the transfer of IXUs, for managing transfer queues, and for changing attributes of specified IXUs. Due to the attribute system, the MDTS is able to operate independently, without a direct user control. According to user specifications, expressed by attributes of IXUs, the MDTS can make its own decisions about invoking operations when conditions are favorable, about postponing transfers when conditions deteriorate, about 
trying to recover from failures, and about cancelling operations. In certain circumstances, the MDTS can even be allowed to make telephone calls.

In the data transport layer, the standard TCP/IP protocol stack is replaced with the Mowgli Data Channel Service (MDCS), which takes care of transmitting data over the wireless link between the mobile node and the MCH. All communication above the data transport layer uses the transport services offered by the MDCS. The MDCS is designed to cope with the special characteristics of the cellular telephone links. The communication is based on data channels with a priority-based scheduling system. Furthermore, each data channel has a set of additional attributes for controlling the behavior of the channel in case of exceptional events. The programming interface to the MDCS is very similar to the standard BSD socket interface. A detailed description of the MDCS can be found in Kiiskinen et al. (1996).

\section{BENEFITS OF THE MOWGLI APPROACH}

The mediator-based architecture of Mowgli affects the behavior of applications in several ways. Below we briefly summarize various benefits observed in experiments with Mowgli prototypes.

\subsection{Working in normal conditions}

The performance of distributed applications is essentially improved. The major benefits can be attributed to the agent-proxy team which makes it possible to divide the end-toend data communication path into two autonomous parts. The agent-proxy team can also exploit the application semantics leading, for example, to type-specific data compression and to application-specific error recovery. We have also implemented several technical improvements including reduction of round trips, avoidance of unnecessary data transmissions, error recovery from short-term disconnections, and compression of headers. The increase in performance is remarkable as reported in Liljeberg et al. $(1995,1996)$ and in Kiiskinen et al. (1996).

In a wireless WAN environment the user's possibility to control the communication is important. We allow the user to specify the order of data transmissions, to query the expected transmission times, and to decide if the data should be sent immediately. We have also allowed the user to delegate (with given advice) the decision making to the agent-proxy team.

\subsection{Working in disconnected mode}

There are three primary reasons why the user may want to work without a connection. Firstly, the wireless link is usually quite expensive. Secondly, the link quality may temporarily be below a required Quality-of-Service level. Thirdly, the user may be in an area not covered by the cellular telephone system.

In the disconnected mode a Mowgli agent in the mobile node can take the role of the remote component in the application. The basic principle is to transform the user data into Information eXchange Units. When the connection is later established, the Mowgli Data Transfer Service takes care of transferring the IXUs without any explicit user intervention. 
The agent-proxy approach gives rise to functionality that may, in the future, turn out to be the most important one: The proxy can take the role of the mobile component and proceed autonomously. For example, the agent delegates time-consuming operations to the proxy to be executed asynchronously. The independently working proxy can control remote operations and receive incoming messages. If needed, it can reestablish the connection with the mobile node.

\subsection{Working in almost disconnected mode}

Traditionally the nodes in distributed systems work either in a connected mode or in a disconnected mode. The mobile telephone systems give another possibility: Components of the application will only connect to each other at the moment when cooperation is really needed. Typical examples include cache validation and notification of incoming messages.

An alternative usage for proxies: An application-specific proxy may act as an intelligent agent for the mobile node. It works independently, in a disconnected mode, to fulfill a task specified by the mobile application. For example, it may filter incoming data, and when something of interest arrives, it sends a notification to the mobile node.

The implementation of notifications can be based on the Short Message Service of GSM, which is a low-bandwidth but light-weight form of communication over the wireless link. An important implication is that the connection setup can be implemented in an almost transparent manner: The agent and the proxy communicate according to predefined rules.

\subsection{Working in changing conditions}

The disconnected operations can be exploited further. The user may be willing to specify minimum conditions under which the data transfer should take place. For example, large files are transferred only during cheap night time or during periods of high link quality, but urgent messages are always sent as soon as possible. The MDTS invokes the operations when the specified conditions are satisfied. In other words, the operations of the MDTS can be controlled like guarded commands.

\section{ESSENTIALS OF MOWGLI}

The original challenge in the Mowgli project was to combine two data communication systems of very different characteristics under a single control. This was accomplished through a mediator, consisting of an agent-proxy team and the associated infrastructure.

The role of the agent-proxy team is pronounced: Together the agent and proxy form a model for a distributed intelligent agent. This agent is "Janus-faced" in two ways. Horizontally it presents the client-interface on the fixed-net side and the server-interface on the mobile node, or vice versa. Vertically, it knows both the application semantics and the problems in wireless communication. This general approach gives a rich choice of opportunities for the application developer:

- hide or show,

- make the control "goal-based" or "explicit",

- solve the conflicts between the user's wishes and skills - or let the user do it. 
An intelligent agent can take several different roles. The possibilities include the roles of an advisor, a filter, a booster, and a representative.

Today the research in mobility is primarily concentrated on the problems of physical mobility: The systems perceive how the nodes move. In the Mowgli environment the cellular telephone system hides certain issues in the terminal mobility like paging, hand-overs, and location updates. Therefore, we have been able to focus on the next set of problems: when to connect, how to control disconnected agents, how to handle the multiple-copyupdate problem when the peers are only probably accessible, and - in general - how to improve the dependability of applications comprising wireless data communications.

Traditionally middleware solutions have been regarded as a promising approach in implementing distributed applications on heterogeneous platforms. According to our experience we would like to extend the concept: when implementing distributed applications in heterogeneous data communication environments the middleware should be replaced by a functionally more powerful "mediatorware".

\section{ACKNOWLEDGEMENTS}

This work was carried out as a part of the Mowgli research project funded by Digital Equipment Corporation, Nokia Mobile Phones, Nokia Telecommunications, and Telecom Finland. The authors are thankful to the rest of the Mowgli team, particularly Heikki Helin, Petteri Kaskenpalo, Jani Kiiskinen and Mika Liljeberg, for the fruitful comments and discussions during the Mowgli project as well as during the preparation of this paper. Last but not least the authors want to express their gratitude to the software engineering student groups who have participated in the implementation of the system.

\section{REFERENCES}

Alanko, T., Kojo, M., Laamanen, H., Liljeberg, M., Moilanen, M. and Raatikainen, K. (1994) Measured Performance of Data Transmission over Cellular Telephone Networks. Computer Communications Review, 24, 5, 24-44.

Bakre, A. and Badrinath, B.R. (1995) I-TCP: Indirect TCP for Mobile Hosts, in Proceedings of the IEEE 15th International Conference on Distributed Computer Systems, IEEE Computer Society, Los Alamitos.

Balakrishnan, H., Seshan, S., Amir, E. and Katz, R. (1995) Improving TCP/IP Performance over Wireless Networks, in Proceedings of the First ACM International Conference on Mobile Computing and Networking (Mobicom '95), ACM, New York.

Kiiskinen, J., Kojo, M., Liljeberg, M. and Raatikainen, K. (1996) Data Channel Service for Wireless Telephone Links, in Proceedings of the Second International Mobile Computing Conference, National Chiao Tung University, Hsinchu.

Kojo, M., Raatikainen, K. and Alanko, T. (1994) Connecting Mobile Workstations to the Internet over a Digital Cellular Telephone Network. Report C-1994-39, University of Helsinki, Department of Computer Science. Revised version in Mobile Computing (eds. Imieliński, T. and Korth, H.F. 1996), Kluwer, Boston.

Liljeberg, M., Alanko, T., Kojo, M., Laamanen, H. and Raatikainen, K. (1995) Optimizing World-Wide Web for Weakly-Connected Mobile Workstations: An Indirect Approach, 
in Proceedings of the 2nd International Workshop on Services in Distributed and Networked Environments (SDNE), IEEE Computer Society, Los Alamitos.

Liljeberg, M., Helin, H., Kojo, M. and Raatikainen, K. (1996) Enhanced Services for World-Wide Web in Mobile WAN Environment, to appear in Proceedings of the 3rd International Conference on Communicating by Image and Multimedia (Image'Com 96), ADERA, Bordeaux.

Rahnema, M. (1993) Overview of the GSM System and Protocol Architecture. IEEE Communication Magazine, 31, 4, 92-100.

Yavatkar, R. and Bhagawat, N. (1994) Improving End-to-End Performance of TCP over Mobile Internetworks, in Proceedings of the IEEE Workshop on Mobile Computing Systems and Applications, IEEE Computer Society, Los Alamitos.

\section{BIOGRAPHY}

Timo Alanko received in 1983 his Ph.D. (Computer Science) from the University of Helsinki. He is an Assistant Professor in Computer Science at the University of Helsinki; currently the project leader of Mowgli. His research interests include distributed operating systems, mobile computing, and performance analysis. Markku Kojo received in 1995 his M.Sc. (Computer Science) from the University of Helsinki. Currently he is Ph.D. student in Computer Science at the University of Helsinki. His research interests include mobile computing, data communications, and distributed systems. Heimo Laamanen received in 1982 his M.Sc. (Computer Science) from the University of Helsinki. Currently he is researcher in Digital Equipment Corporation and Ph.D. student in Computer Science at the University of Helsinki. His research interests include nomadic computing, wireless data communications, and distributed systems. Kimmo Raatikainen received in 1990 his Ph.D. (Computer Science) from the University of Helsinki. Since 1990 he has been Assistant Professor in Computer Science at the University of Helsinki. He is a member of IFIP TC6 Special Interest Group of Intelligent Networks. His research interests include nomadic computing, telecommunications software architectures, and real-time databases. Matti Tienari received in 1962 his Ph.D. (Mathematics) from the University of Helsinki. Since 1969 he has been Full Professor in Computer Science at the University of Helsinki; currently Chairman of the Department. He has been the representative of Finland at IFIP General Assembly 1987-96, IFIP Trustee 1989-95 and member of IFIP WG 6.1 (Architecture and Protocols for Computer Networks). His research interests include modeling of concurrency, computer networks, and distributed systems. 